\title{
Depletion layer and contact capacitance in non-uniformly doped semiconductors
}

\author{
A Shik ${ }^{1}$, H E Ruda ${ }^{1}$, D Pelinovsky ${ }^{2}$ and W Craig ${ }^{2}$ \\ ${ }^{1}$ Energenius Centre for Advanced Nanotechnology, University of Toronto, Toronto, \\ Ontario M5S 3E4, Canada \\ ${ }^{2}$ Department of Mathematics, McMaster University, Hamilton, Ontario L8S 4K1, Canada
}

Received 20 May 2002

Published 4 November 2002

Online at stacks.iop.org/JPhysD/35/2988

\begin{abstract}
We consider the dependence of the capacitance $C$ of the semiconductor depletion layer versus the contact bias $V$. When the concentration of doping impurities varies in the plane parallel to the contact, we show that the standard Schottky dependence $C^{-2}(V)$ is no longer a straight line. We compute the effective concentration $N_{\text {eff }}$ from the slope of the dependence $C^{-2}(V)$ by using the perturbation and variation methods and compare $N_{\text {eff }}$ with previous theoretical and experimental data.
\end{abstract}

\section{Introduction}

The concentration of doping impurities $N$ in a reverse biased Schottky contact is often deduced from the slope of the capacitance-voltage characteristic (CVC). If the doping is nonuniform and varies along the direction normal to the sample surface provided with a Schottky contact ( $z$-axis), the effective concentration can be recovered from the experimental CVC measurements [1]. However, in practice, semiconductors contain different kinds of inhomogeneities so that the impurity concentration varies not only along the $z$-axis but in the $(x, y)$-plane as well. These inhomogeneities are especially pronounced in strongly compensated, irradiated and polycrystalline semiconductors, and they can change dramatically their transport and photoelectric properties [2].

In this paper we discuss the CVC properties of semiconductors with doping concentration varying in the plane parallel to the surface. We answer the following key question: if standard CVC measurements are performed in an inhomogeneous semiconductor with spatially varying doping level $N(\boldsymbol{r})$, how is the effective concentration $N_{\text {eff }}$ (defined from the slope of CVC) related to $N(\boldsymbol{r})$ ? The question is especially interesting when different experimental methods of concentration measurements, which give the same result in homogeneous samples, result in dramatically different measurement of $N_{\text {eff }}$ for the same inhomogeneous sample. For instance, in the case of high-frequency and Hall effect measurements, $N_{\text {eff }}$ is close to the spatially averaged $\overline{N(\boldsymbol{r})}$, which in strongly non-uniform samples may be up to several orders of magnitude higher than the effective (percolation) concentration determining the static conductivity [2].
The structure of the paper is as follows. In section 2, we give a mathematical formulation of the problem and discuss preliminary estimates on how the effective concentration depends on the characteristic parameters of the inhomogeneity, including the mean and standard deviation of fluctuations, their correlation length and the effective thickness of the depletion layer. In section 3 , we solve the problem using the perturbation series, provided the standard deviation of the fluctuations is small. In section 4 , we give an independent analysis of the same problem by using a variational method, when the inhomogeneity has a stepwise constant profile. Section 5 concludes the paper by comparing our results with experimental data.

\section{Formulation of the problem and general estimates}

The problem of finding the CVC for an inhomogeneous semiconductor can be formulated as a nonlinear boundaryvalue problem for the Poisson equation. We assume that the semiconductor with an inhomogeneous doping level $N(\boldsymbol{r})$ occupies the half-space $z>0$. The plane $z=0$ is an equipotential metallic contact with some applied voltage $V$ such that

$$
\varphi(z=0)=V,
$$

whereas in the bulk of semiconductor the potential vanishes: $\varphi(z \rightarrow \infty)=0$. These requirements represent boundary conditions for the Poisson equation $\Delta \varphi=(4 \pi e / \varepsilon)[n(\varphi)-$ $N(\boldsymbol{r})$ ], where $e$ is the electron charge, $\varepsilon$ is the dielectric susceptibility, and $n(\varphi)$ is the concentration of free electrons depending on the local value of potential. 
The presence of the nonlinear function $n(\varphi)$ makes the problem in its general form extremely complicated. We will consider the case of a strong depletion (which for n-type semiconductors corresponds to large negative $V$ ), where the problem can be essentially simplified by using the so-called Schottky approximation, which is adequate if $e|V|$ considerably exceeds the thermal energy $k T$. The latter assumes that in all points with $\varphi<0$, the concentration $n(\varphi)$ is negligible. Let the surface $z=\eta(x, y)$ be the boundary of this completely depleted region $E=\{(x, y, z): \varphi \leqslant 0, z \geqslant 0\}$ and make the principal assumption that $n(\varphi)=0$ in $E$. By the maximum principle, we conclude that $\varphi=0$ in $E$ and, in particular,

$$
\varphi(z=\eta(x, y))=0 .
$$

As a result, instead of solving the Poisson equation in the whole half-space, we may restrict ourselves only to the region $0<z<\eta(x, y)$ where the linear Poisson equation,

$\Delta \varphi=\frac{\partial^{2} \varphi}{\partial x^{2}}+\frac{\partial^{2} \varphi}{\partial y^{2}}+\frac{\partial^{2} \varphi}{\partial z^{2}}=-e^{*} N(x, y, z), \quad e^{*}=\frac{4 \pi e}{\varepsilon}$,

is to be solved with the boundary conditions equations (1) and (2). The function $\eta(x, y)$ defines the boundary of the completely depleted region. Since the function $\eta(x, y)$ is unknown, an additional boundary condition makes the problem well-posed. If $\varphi=0$ for all $z \geqslant \eta(x, y)$ and $\varphi$ is smooth, then the normal derivative of $\varphi$ vanishes at the surface, which results in the following boundary condition on the free boundary:

$$
\frac{\partial \varphi}{\partial z}(z=\eta(x, y))=0 .
$$

The resulting free surface $z=\eta(x, y)$ is the surface of zero potential and zero electric field flux.

Once the Poisson equation with the given boundary conditions is solved and $\eta(x, y)$ determined, the capacitance $C$ of the structure can be easily found. By definition, $C=$ $\mathrm{d} Q / \mathrm{d}|V|$, where $Q=Q(V)$ is the electric charge per unit area depending on the applied voltage $V$ :

$$
Q=\frac{e^{*}}{S} \int_{S} \mathrm{~d} x \mathrm{~d} y \int_{0}^{\eta(x, y)} N(x, y, z) \mathrm{d} z
$$

with $S$ being a region in the $(x, y)$-plane with dimensions considerably exceeding the typical size $L$ of inhomogeneities of $N(\boldsymbol{r})$. For uniform doping, $N(\boldsymbol{r})=\bar{N}=$ const., the problem has a well-known parabolic solution:

$\varphi=\Phi_{0}(z)=-\frac{e^{*} \bar{N}}{2}\left(\eta_{0}-z\right)^{2}, \quad \eta=\eta_{0}=\sqrt{\frac{2|V|}{e^{*} \bar{N}}}$,

where $V<0$. The parabolic solution results in the classical formula for CVC:

$$
C_{0}=\frac{\mathrm{d} Q_{0}}{\mathrm{~d}|V|}=e^{*} \bar{N} \frac{\mathrm{d} \eta_{0}}{\mathrm{~d}|V|}=\left(\frac{e^{*} \bar{N}}{2|V|}\right)^{1 / 2} .
$$

The goal of this paper is to study possible modifications of CVC due to spatial inhomogeneity of the doping concentration in the plane parallel to the semiconductor surface, described by $N=N(x, y)$. In particular, we compute an effective concentration defined as:

$$
N_{\mathrm{eff}}=\frac{2}{e^{*}}|V| C^{2}(V) .
$$

Measured from experiment, the effective concentration $N_{\text {eff }}$ is typically interpreted to be the doping level of the semiconductor. In uniform samples, it is true since $N_{\text {eff }}=$ $\bar{N}$ and $N_{\text {eff }}$ is independent of $V$. In the presence of inhomogeneities, the effective concentration $N_{\text {eff }}$ defined in equation (8) may depend on the mean value $\bar{N}$ and the variance $\overline{(N-\bar{N})^{2}}$ of $N=N(\boldsymbol{r})$, its correlation length $L$ and the average thickness of the depletion layer $\eta_{0}$ (i.e. on the applied voltage $V$ ).

Some general results can be derived from elementary considerations (see also [3, 4]). For $L \ll \eta_{0}$, spatial fluctuations of concentration $N(\boldsymbol{r})$ are not relevant at the length scale $\eta_{0}$, so that the screening is determined by an average concentration: $N_{\text {eff }}=\bar{N}$. In the opposite limit $L \gg \eta_{0}$ the depletion layer can be considered as the parallel array of microscopically, uniformly doped regions, with capacitances proportional to the square root of the local $N$ and, hence, $N_{\text {eff }}=(\overline{\sqrt{N}})^{2}$. Since, for any inhomogeneity, $(\overline{\sqrt{N}})^{2}<$ $\bar{N}$, we may expect that for inhomogeneities with the same mean value and standard deviation of fluctuations $N(\boldsymbol{r})$, the effective concentration $N_{\text {eff }}$ decreases with their correlation length $L$ and increases with the depleting voltage $V$, due to the increase in the ratio $\eta_{0} / L$. We confirm these general estimates by analysing the perturbation theory and variational methods for the problem equation (3) with the boundary conditions equations (1), (2) and (4) and show that the effective

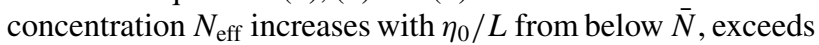
the value $\bar{N}$ at a finite $\eta_{0} / L$ and approaches $\bar{N}$ from above in the limit of large $\eta_{0} / L$. In our further calculations we restrict ourselves to the case of one-dimensional fluctuations, $N=N(x)$, and reduce the problem to a planar geometry: $\varphi=\varphi(x, z)$ and $\eta=\eta(x)$.

\section{Perturbation series results}

Let $N(x)=\bar{N}+\epsilon M(x)$, where non-uniform perturbation $M(x)$ has the properties: $M(x+2 L)=M(x), \bar{M}=0$, and $\bar{M}^{2}=1$. Here and henceforth, the overline denotes the mean value of a function on the interval $x \in[-L, L]$. The parameter

$$
\epsilon=\sqrt{\overline{(N(x)-\bar{N})^{2}}}
$$

measures a small standard deviation of the perturbation term $M(x)$. The periodic function $M(x)$ can be expanded into complex Fourier series:

$$
\begin{gathered}
M(x)=\sum_{n=-\infty}^{\infty} m_{n} \mathrm{e}^{\mathrm{i} k_{n} x}, \quad k_{n}=\frac{\pi n}{L}, \\
m_{n}=\overline{m_{-n}}=\frac{1}{2 L} \int_{-L}^{L} M(x) \mathrm{e}^{-\mathrm{i} k_{n} x} \mathrm{~d} x
\end{gathered}
$$

with the Fourier coefficients $m_{n}$ satisfying the constraints: $m_{0}=0$ and $\sum_{n=-\infty}^{\infty}\left|m_{n}\right|^{2}=1$. Solution of the problem, equations (1)-(4), can be found in a formal perturbation series: $\varphi(x, z)=\Phi_{0}(z)+\epsilon \Phi_{1}(x, z)+\epsilon^{2} \Phi_{2}(x, z)+\mathrm{O}\left(\epsilon^{3}\right)$,

$$
\eta(x)=\eta_{0}+\epsilon \eta_{1}(x)+\epsilon^{2} \eta_{2}(x)+\mathrm{O}\left(\epsilon^{3}\right),
$$


where the zero-order term is given by equation (6), while the first-order and second-order terms for the potential $\varphi(x, z)$ are to be determined from the equations

$$
\begin{gathered}
\Delta \Phi_{1}=-e^{*} M(x), \quad \Phi_{1}(x, 0)=0, \\
\Phi_{1}\left(x, \eta_{0}\right)=0, \\
\Delta \Phi_{2}=0, \quad \Phi_{2}(x, 0)=0, \\
\Phi_{2}\left(x, \eta_{0}\right)=-\frac{e^{*} \bar{N}}{2} \eta_{1}^{2}(x) .
\end{gathered}
$$

The terms for the potential are then used to find the depletion layer profile:

$$
\begin{gathered}
\eta_{1}=\frac{1}{e^{*} \bar{N}} \frac{\partial \Phi_{1}}{\partial z}\left(x, \eta_{0}\right), \\
\eta_{2}=-\frac{M(x)}{\bar{N}} \eta_{1}(x)+\frac{1}{e^{*} \bar{N}} \frac{\partial \Phi_{2}}{\partial z}\left(x, \eta_{0}\right) .
\end{gathered}
$$

Solutions of the first-order problem, equations (12) and (14), can be obtained from the Fourier series expansions:

$$
\begin{aligned}
\Phi_{1}= & -e^{*} \sum_{n=-\infty}^{\infty} \frac{m_{n}}{k_{n}^{2}}\left[\cosh k_{n} z-1\right. \\
& \left.+\frac{\sinh k_{n} z}{\sinh k_{n} \eta_{0}}\left(1-\cosh k_{n} \eta_{0}\right)\right] \mathrm{e}^{\mathrm{i} k_{n} x}, \\
\eta_{1} & =\sum_{n=-\infty}^{\infty} \frac{m_{n}\left(1-\cosh k_{n} \eta_{0}\right)}{\bar{N} k_{n} \sinh k_{n} \eta_{0}} \mathrm{e}^{\mathrm{i} k_{n} x} .
\end{aligned}
$$

Solution of the second-order problem equation (13) has the form

$$
\Phi_{2}=-\frac{e^{*} \bar{N}}{2} \sum_{n=-\infty}^{\infty} a_{n} \frac{\sinh k_{n} z}{\sinh k_{n} \eta_{0}} \mathrm{e}^{\mathrm{i} k_{n} x}
$$

where $a_{n}$ are the Fourier coefficients of $\eta_{1}^{2}(x)$ :

$a_{n}=\sum_{l=-\infty}^{\infty} \frac{m_{l} \bar{m}_{l-n}\left(1-\cosh k_{l} \eta_{0}\right)\left(1-\cosh k_{l-n} \eta_{0}\right)}{\bar{N}^{2} k_{l} k_{l-n} \sinh k_{l} \eta_{0} \sinh k_{l-n} \eta_{0}}$.

We compute the charge $Q=Q(V)$ by averaging $\eta(x) N(x)$ :

$$
Q=\frac{e^{*}}{2 L} \int_{-L}^{L} \eta(x) N(x) \mathrm{d} x=Q_{0}+\epsilon^{2} Q_{2}+\mathrm{O}\left(\epsilon^{4}\right),
$$

where odd terms of the series in $\epsilon$ have zero mean and therefore do not contribute to $Q$, while $Q_{0}=e^{*} \eta_{0} \bar{N}$ and $Q_{2}$ takes the form

$Q_{2}=\frac{e^{*}}{2 L} \int_{-L}^{L} \mathrm{~d} x\left(\eta_{2} \bar{N}+\eta_{1} M\right)=\frac{1}{2 L} \int_{-L}^{L} \frac{\partial \Phi_{2}}{\partial z}\left(x, \eta_{0}\right) \mathrm{d} x$, or, explicitly,

$$
Q_{2}=-\frac{e^{*} \bar{N} a_{0}}{2 \eta_{0}}=-\sum_{n=-\infty}^{\infty} \frac{e^{*}\left|m_{n}\right|^{2}\left[1-\cosh k_{n} \eta_{0}\right]^{2}}{2 \bar{N} k_{n}^{2} \eta_{0} \sinh ^{2} k_{n} \eta_{0}} .
$$

The resulting CVC defined from $C=\mathrm{d} Q / \mathrm{d}|V|$ can be represented in the form equation (8), where the effective concentration

$$
\begin{aligned}
N_{\text {eff }} & =\bar{N}\left[1+\epsilon^{2} \sum_{n=-\infty}^{\infty}\right. \\
& \left.\times \frac{\left|m_{n}\right|^{2}\left[1-\cosh k_{n} \eta_{0}\right]^{2}\left[\sinh k_{n} \eta_{0}-2 k_{n} \eta_{0}\right]}{\bar{N}^{2} k_{n}^{2} \eta_{0}^{2} \sinh ^{3} k_{n} \eta_{0}}+\mathrm{O}\left(\epsilon^{4}\right)\right] .
\end{aligned}
$$

The asymptotic limits of small and large $\eta_{0} / L$ correspond to the general predictions described in section 2. Consider, for example, a finite Fourier series in equation (9) for $|n| \leqslant n_{\max }$ and take the limit $k_{n} \eta_{0}=\pi n \eta_{0} / L \rightarrow 0$ for any $n \leqslant n_{\max }$. Then, equation (21) reduces to the simple formula:

$$
\begin{aligned}
& N_{\text {eff }}\left(\bar{N}, \epsilon, L, \eta_{0}\right)=\bar{N}\left[1-\frac{\epsilon^{2}}{4 \bar{N}^{2}} \sum_{n=-n_{\max }}^{n_{\max }}\left|m_{n}\right|^{2}\right. \\
& \left.\quad+\mathrm{O}\left(\frac{\epsilon^{2} \eta_{0}^{2}}{L^{2}}, \epsilon^{4}\right)\right] \leqslant \bar{N} .
\end{aligned}
$$

Since $\sum_{n=-\infty}^{\infty}\left|m_{n}\right|^{2}=1$, then

$$
(\sqrt{\sqrt{\bar{N}+\epsilon M(x)}})^{2}=\bar{N}\left[1-\frac{\epsilon^{2}}{4 \bar{N}^{2}}+\mathrm{O}\left(\epsilon^{4}\right)\right],
$$

and it follows from equation (22) that $N_{\text {eff }}=(\overline{\sqrt{N(x)}})^{2}<\bar{N}$ in the limit of small $\eta_{0} / L$. In the opposite limit of large $\eta_{0} / L$, all terms of the complex Fourier series equation (9) can be uniformly simplified as

$$
\begin{aligned}
& N_{\text {eff }}\left(\bar{N}, \epsilon, L, \eta_{0}\right)=\bar{N}\left[1+\frac{\epsilon^{2} L^{2}}{\bar{N}^{2} \pi^{2} \eta_{0}^{2}} \sum_{n=-\infty}^{\infty} \frac{\left|m_{n}\right|^{2}}{n^{2}}\right. \\
& \left.\quad+\mathrm{O}\left(\frac{\epsilon^{2} L^{4}}{\eta_{0}^{2}}, \epsilon^{4}\right)\right] \geqslant \bar{N} .
\end{aligned}
$$

Again, the perturbation series confirm the general prediction that $N_{\text {eff }}=\bar{N}$ in the limit of large $\eta_{0} / L$. A new property, which is not predicted from simple qualitative arguments, consists in the fact that the effective concentration $N_{\text {eff }}$ approaches $\bar{N}$ from above, i.e. $N_{\text {eff }} \geqslant \bar{N}$ for large $\eta_{0} / L$. It means that the dependence of $N_{\text {eff }} / \bar{N}$ versus $\eta_{0} / L$ has a non-monotonic character.

We illustrate the behaviour of $N_{\text {eff }}$ for an arbitrary spatial scale $\eta_{0} / L$ by considering the piecewise constant profile for the doping concentration: $N(x)=N_{+}$for $0<x<L$ and $N(x)=N_{-}$for $-L<x<0$. We set $N_{+}>N_{-}$for convenience and define

$$
\bar{N}=\frac{N_{+}+N_{-}}{2}, \quad \epsilon=\frac{N_{+}-N_{-}}{2} .
$$

Then, the perturbation term is $M(x)=\operatorname{sign}(x)$ with the Fourier series equation (9) with $m_{n}=2 /(i \pi n)$ for odd $n$ and $m_{n}=0$ for even $n$. The ratio of the effective and mean concentrations $N_{\text {eff }} / \bar{N}$ versus $\eta_{0} / L$ is shown on figure 1 by the dotted curve. All properties described above are well illustrated by this dependence.

\section{Variational results}

In the previous section, we described perturbation results that show the main tendencies in the dependence of the effective concentration $N_{\text {eff }}$ on the spatial scale of inhomogeneities $\eta_{0} / L$. However, the perturbation results may not be relevant for many applications since they are limited by low-amplitude fluctuations of the doping concentration $N=N(\boldsymbol{r})$. Here, we develop a variational approach to the problem in order to study inhomogeneities of arbitrary amplitude.

We assume again that the doping profile $N(x)$, the Schottky surface $\eta(x)$, and the potential $\varphi(x, z)$ are periodic in $x$ with period $2 L$. Solution of the Poisson equation, 


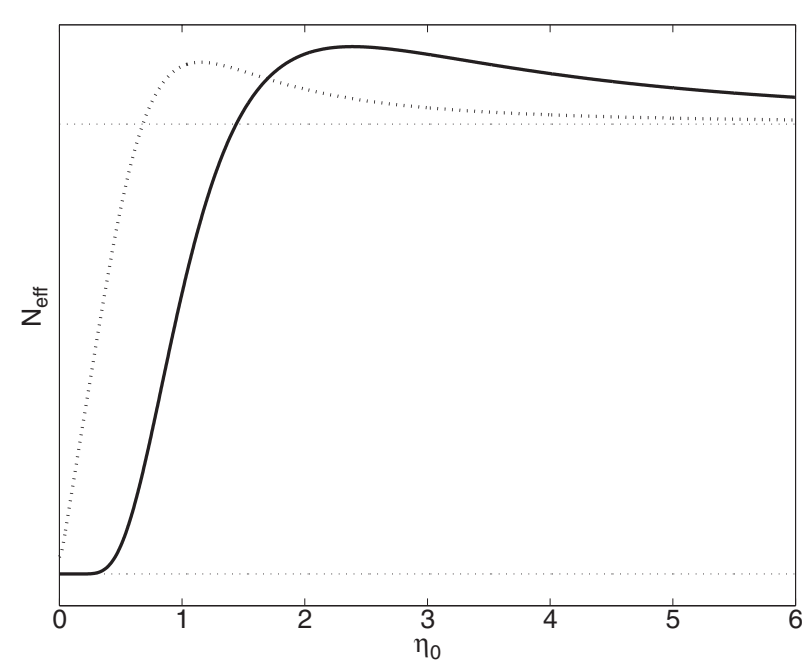

Figure 1. The dependence of $N_{\text {eff }} / \bar{N}$ versus $\eta_{0}$ for a piecewise approximation of the doping concentration with $N_{+}=1.25 \bar{N}$ and $N_{-}=0.75 \bar{N} . \eta_{0}$ is measured in units of $L$. The upper horizontal dotted line corresponds to $N_{\text {eff }}=\bar{N}$, the lower one to $N_{\text {eff }}=(\overline{\sqrt{N}})^{2}$. The dotted curve is the result of the perturbation theory, while the solid curve gives the result of the variational method.

equation (3), can be derived as a variational (minimization) problem for the electrostatic energy functional:

$$
\begin{gathered}
W[\varphi, \eta]=\int_{-L}^{L} \mathrm{~d} x \int_{0}^{\eta(x)} \mathrm{d} z\left[\frac{1}{2}\left(\frac{\partial \varphi}{\partial x}\right)^{2}+\frac{1}{2}\left(\frac{\partial \varphi}{\partial z}\right)^{2}\right. \\
\left.-e^{*} N(x) \varphi\right] .
\end{gathered}
$$

An arbitrary variations of $W[\varphi, \eta]$ in $\varphi$ and $\eta$ vanish if $\varphi(x, z)$ and $\eta(x)$ satisfy the Poisson equation, equation (3), with the boundary conditions equations (1), (2) and (4). We consider the following class of trial functions for the potential

$$
\varphi=V \frac{[\eta(x)-z]^{2}}{\eta^{2}(x)}, \quad \eta(x)>0 .
$$

The trial function equation (26) satisfies all the boundary conditions equations (1), (2) and (4) but does not solve the Poisson equation, equation (3), unless $N(x)$ and $\eta(x)$ are constant in $x$. Nonetheless, we recover an approximation to the free surface $\eta(x)$ by minimizing the energy functional equation (25) with the trial function equation (26). After substitution of equation (26), the functional takes the form

$$
W[\eta]=\int_{-L}^{L} \mathrm{~d} x\left[\frac{V^{2}\left(\eta^{\prime}\right)^{2}}{15 \eta}+\frac{2 V^{2}}{3 \eta}+\frac{e^{*}|V|}{3} N(x) \eta\right],
$$

and its minimization results in the differential equation for $\eta(x)$,

$$
\frac{2}{5 \eta} \eta^{\prime \prime}-\frac{1}{5 \eta^{2}}\left(\eta^{\prime}\right)^{2}+\frac{2}{\eta^{2}}=\frac{e^{*} N(x)}{|V|},
$$

with the periodic boundary conditions $\eta(x)=\eta(x+2 L)$.

We use again the piecewise approximation of the doping concentration $N(x)$ such that $N(x)=N_{+}$for $0<x<L$ and $N(x)=N_{-}$for $-L<x<0$. This allows us to integrate equation (28) once:

$$
H_{ \pm}=\frac{1}{10 \eta}\left(\frac{\mathrm{d} \eta}{\mathrm{d} x}\right)^{2}-\frac{1}{\eta}-\frac{\eta}{\eta_{ \pm}^{2}}
$$

where $\eta_{ \pm}=\left(2|V| / e^{*} N_{ \pm}\right)^{1 / 2}$. Periodic solutions of equation (28) with this piecewise constant function $N(x)$ satisfy the symmetry conditions $\eta^{\prime}( \pm L / 2)=0$. We denote $\eta_{0}^{ \pm}=\eta( \pm L / 2)$ and express the constants $H_{ \pm}$in terms of $\eta_{0}^{ \pm}$and $\eta_{ \pm}$. Then, the problem, equation (29), reduces to integration of two first-order equations:

$$
\left(\frac{\mathrm{d} \eta}{\mathrm{d} x}\right)^{2}=\frac{10}{\eta_{ \pm}^{2}}\left(\eta-\eta_{0}^{ \pm}\right)\left(\eta-\frac{\eta_{ \pm}^{2}}{\eta_{0}^{ \pm}}\right)
$$

with the boundary conditions $\eta( \pm L / 2)=\eta_{0}^{ \pm}$. At the interface $x=0$ between two solutions, we require continuity of $\eta(0)$ and $\eta^{\prime}(0)$. These matching conditions define parameters $\eta_{0}^{ \pm}$of the solutions. If $N_{+}>N_{-}$, then $\eta_{+}<\eta_{-}$. Solutions of this problem can be viewed qualitatively by using the phase plane for $\left(\eta, \eta^{\prime}\right)$. The points $\eta=\eta_{ \pm}$are critical (saddle) points of the dynamical system equation (28) with $N(x)=N_{ \pm}$. They are the only critical points in the right-half-plane of $\eta(x)>0$ and it can be shown that there exists a unique periodic solution $\eta(x)$ that is a decreasing function of $x$ for $x \in[-L / 2, L / 2]$ between $\eta_{-}>\eta_{0}^{-} \geqslant \eta(x) \geqslant \eta_{0}^{+}>\eta_{+}$.

More quantitatively, the differential equations, equation (30), have the exact analytical solutions

$$
\eta(x)=\frac{1}{2}\left(\eta_{0}^{ \pm}+\frac{\eta_{ \pm}^{2}}{\eta_{0}^{ \pm}}\right)+\frac{1}{2}\left(\eta_{0}^{ \pm}-\frac{\eta_{ \pm}^{2}}{\eta_{0}^{ \pm}}\right) \cosh \frac{\sqrt{10}}{\eta_{ \pm}}\left(x \mp \frac{L}{2}\right)
$$

Matching them at $x=0$ with $\eta(0)$ and $\eta^{\prime}(0)$ being continuous, we derive a system of two algebraic equations for $\eta_{0}^{ \pm}$. Once the system for $\eta_{0}^{ \pm}$is solved, the charge $Q=Q(V)$ can be computed from the formula

$$
\begin{aligned}
Q= & \frac{e^{*} N_{+}}{4}\left(\eta_{0}^{+}+\frac{\eta_{+}^{2}}{\eta_{0}^{+}}\right)+\frac{e^{*} N_{-}}{4}\left(\eta_{0}^{-}+\frac{\eta_{-}^{2}}{\eta_{0}^{-}}\right) \\
& +\frac{e^{*} N_{+}}{4}\left(\eta_{0}^{+}-\frac{\eta_{+}^{2}}{\eta_{0}^{+}}\right) \frac{\sinh \theta_{+}}{\theta_{+}} \\
& +\frac{e^{*} N_{-}}{4}\left(\eta_{0}^{-}-\frac{\eta_{-}^{2}}{\eta_{0}^{-}}\right) \frac{\sinh \theta_{-}}{\theta_{-}}
\end{aligned}
$$

where $\theta_{ \pm}=\sqrt{10} L /\left(2 \eta_{ \pm}\right)$. The effective concentration $N_{\text {eff }}$ is computed from the derivative $C=\mathrm{d} Q / \mathrm{d}|V|$ according to equation (8). The dependence of $N_{\text {eff }} / \bar{N}$ versus $\eta_{0} / L$ is shown in figure 1 by the solid curve. Both the solid (variational) and dotted (perturbation) curve agree well and demonstrate similar behaviour of the effective concentration: the ratio $N_{\text {eff }} / \bar{N}$ is less than one for small values of $\eta_{0} / L$, greater than one for finite values of $\eta_{0} / L$ and approaches one from above for large values of $\eta_{0} / L$

Figure 2 shows the $\operatorname{CVC} C^{-2}(|V|)$ for one particular example of inhomogeneity. Being compared with the straight line describing CVC in a uniformly doped sample (dotted line), it shows slight deviations in the form of initial sublinear part followed by an inflection point. If we express this dependence in terms of $N_{\text {eff }}$, rather than $C$ (figure 3 ), the deviation becomes much more noticeable.

The asymptotic limits of small and large values of $\eta_{0} / L$ can be computed analytically from the exact solutions equations (31) and (32). In the limit of small values of $\eta_{0} / L$, 


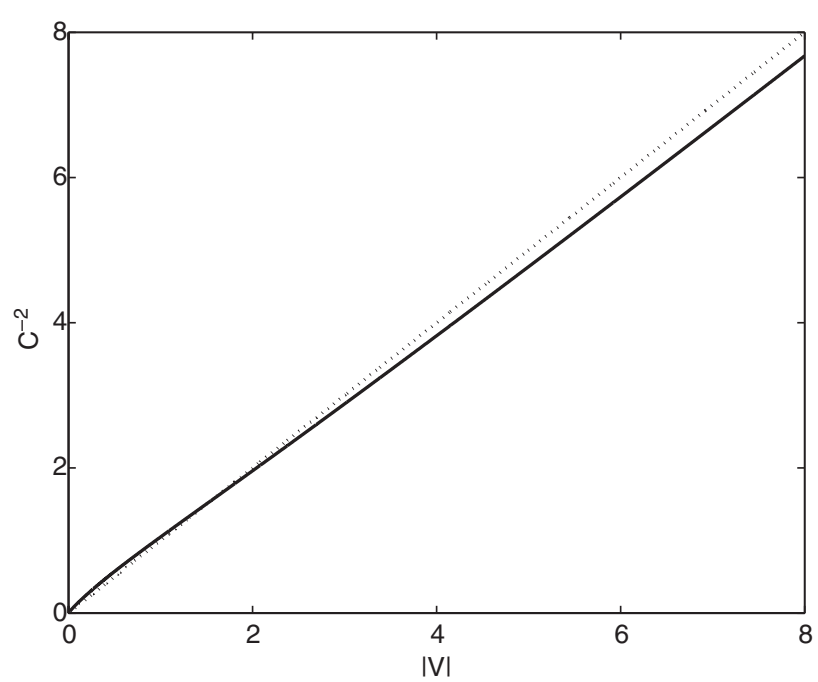

Figure 2. The $\mathrm{CVC}^{-2}(|V|)$ calculated by the variational method for a piecewise approximation of the doping concentration, with $N_{+}=10, N_{-}=0.1$. $C$ is measured in units of $1 / L$ and $V$ is measured in units of $e^{*} \bar{N} L^{2} / 2$. The dotted line corresponds to a uniformly doped sample.

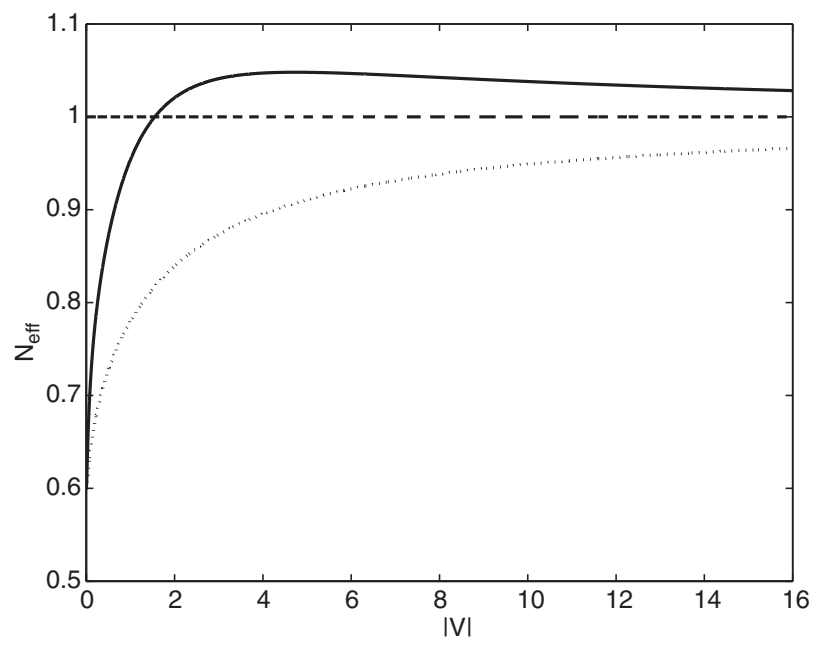

Figure 3. The dependence of $N_{\text {eff }}$ versus $|V|$ for a piecewise approximation of the doping concentration, with $N_{+}=10$ and $N_{-}=0.1$. The solid curve shows $N_{\text {eff }}$ for the differential capacitance $C=\mathrm{d} Q / \mathrm{d}|V|$ and the dotted curve shows $N_{\text {eff }}$ for the static capacitance $C=Q /|V|$. The concentration $N_{\text {eff }}$ is measured in units of $\bar{N}$ and the voltage $V$ is measured in units of $e^{*} \bar{N} L^{2} / 2$.

the solution $\eta(x)$ connects the stable and unstable manifolds of the saddle points $\eta_{ \pm}$, when $\eta_{0}^{ \pm}=\eta_{ \pm}$and

$$
\begin{array}{ll}
\eta=\eta_{-}\left(1+\frac{\eta_{+}-\eta_{-}}{\eta_{+}+\eta_{-}} \mathrm{e}^{\left(\sqrt{10} / \eta_{-}\right) x}\right), & x<0, \\
\eta=\eta_{+}\left(1-\frac{\eta_{+}-\eta_{-}}{\eta_{+}+\eta_{-}} \mathrm{e}^{\left(-\sqrt{10} / \eta_{+}\right) x}\right), & x>0
\end{array}
$$

so that the electric charge $Q$ is given by

$$
Q=\frac{e^{*}}{2}\left(N_{+} \eta_{+}+N_{-} \eta_{-}\right)+\mathrm{O}\left(\frac{\eta_{0}^{2}}{L^{2}}\right)
$$

Using this expression, we confirm the general estimate that

$$
N_{\text {eff }}=\frac{1}{4}\left(\sqrt{N_{+}}+\sqrt{N_{-}}\right)^{2}=(\overline{\sqrt{N}})^{2}<\bar{N}
$$

in the limit of small $\eta_{0} / L$. In the limit of large values of $\eta_{0} / L$, we find an asymptotic solution for $\eta_{0}^{ \pm}$in the form

$$
\eta_{0}^{ \pm}=\eta_{0}\left[1+\frac{L^{2}}{\eta_{0}^{2}} \delta_{2}^{ \pm}+\mathrm{O}\left(\frac{L^{4}}{\eta_{0}^{4}}\right)\right],
$$

where $\eta_{0}$ is given by equation (6), and

$$
\begin{gathered}
\delta_{2}^{+}=-\frac{5\left(N_{+}-N_{-}\right)\left(N_{+}+3 N_{-}\right)}{64 \bar{N}^{2}}, \\
\delta_{2}^{-}=\frac{5\left(N_{+}-N_{-}\right)\left(3 N_{+}+N_{-}\right)}{64 \bar{N}^{2}} .
\end{gathered}
$$

As a result, in this limit the electric charge is given by

$$
Q=e^{*} \eta_{0} \bar{N}\left[1-\frac{5 L^{2}}{192 \bar{N}^{2} \eta_{0}^{2}}\left(N_{+}-N_{-}\right)^{2}+\mathrm{O}\left(\frac{L^{4}}{\eta_{0}^{4}}\right)\right] \text {. }
$$

We confirm again the general estimate in the limit of large $\eta_{0} / L$ :

$$
N_{\text {eff }}=\bar{N}\left[1+\frac{5 \epsilon^{2} L^{2}}{24 \bar{N}^{2} \eta_{0}^{2}}+\mathrm{O}\left(\frac{L^{4}}{\eta_{0}^{4}}\right)\right] \geqslant \bar{N},
$$

in the limit of large $\eta_{0} / L$, where $\epsilon$ is defined by equation (24). Formulae equations (23) and (37) are obtained in the same limit of large $\eta_{0} / L$ by the perturbation theory and by the variation method, respectively. They agree well, except for numerical coefficients.

In defining the effective concentration $N_{\text {eff }}$ as the slope of the curve $C^{-2}(|V|)$, we have found that $N_{\text {eff }}$ exceeds the average concentration $\bar{N}$ for large values of $\eta_{0} / L$, if the capacitance is defined as the differential capacitance: $C=\mathrm{d} Q / \mathrm{d}|V|$. However, in the limit of large values of $\eta_{0} / L$, equations (20) and (36) imply that $Q \leqslant e^{*} \eta_{0} \bar{N}$. The phenomenon is that $N_{\text {eff }}>\bar{N}$ not because of large levels of induced charge but rather from rapid variation of this charge with respect to applied voltage. Using an alternative definition of the capacitance $C$ as the static approximation: $C=Q /|V|$, we obtain that the effective concentration equation (8) will be a monotonic function of $\eta_{0} / L$, bounded by $N$ and approaching this value in the limit of large values of $\eta_{0} / L$ (see the dotted curve in figure 3 ).

\section{Discussion and conclusions}

We have shown that the presence of lateral doping inhomogeneities may influence noticeably the CVC of a Schottky depletion layer. General considerations supported by perturbation and variational analysis demonstrate that the standard $C^{-2}(V)$ dependence in non-uniform samples is no longer a straight line. Its slope, for uniform samples inversely proportional to the doping level (see equation (7)), varies from higher values at low $|V|$ to lower values corresponding to $\bar{N}$ at $|V| \rightarrow \infty$, with some minimum in between. Similar characteristics have been experimentally observed in different types of inhomogeneous materials, such as polycrystalline $\mathrm{Ge}$ [5], nanocrystalline $\mathrm{CdS}$ [6], porous $\mathrm{SiC}$ [7], $\mathrm{TiO}_{2}$ [8] and $\mathrm{GaP}$ 
[9], in GaAs subjected to high energy ion irradiation [10] and in InSe(:Er) [11] where the authors themselves point at a possible non-uniformity caused by the surface melting. In particular, the $C^{-2}(V)$ curves of $[6,7,10]$ demostrate a noticeable decrease of slope at large $|V|$ and in [11] a minimum of the slope at intermediate voltages is revealed especially distinctly.

In our calculations we used the Schottky approximation assuming a sharp boundary between the complete depletion and quasi-neutral regions, without any intermediate layer. This approximation is known to be adequate at $e|V| \gg k T$, which is the main assumption in our calculations. This inequality is the standard requirement for the CVC experiments fulfilled in all experimental works cited above.

It is worth noting that another possible type of inhomogeneity in Schottky barriers-fluctuations of the barrier height (the parameter $V$ in our notation) — does not result in noticeable deviation of $C^{-2}(V)$ from the linear dependence (see, e.g. [12]). Thus, the nonlinear character of the dependence of $C^{-2}(V)$ indicates that the inhomogeneities in a Schottky barrier are of a particular type, namely, are produced by spatial variations of the doping level.

In this connection we add that $C^{-2}(V)$ of the type of figure 2 can be observed not only for lateral doping variations described in our paper but also in layered doping inhomogeneity $N(z)$ with $N$ increasing with $z$ (from surface to the bulk). To distinguish between these two types of doping inhomogeneities, the results should be compared with the data on $N_{\text {eff }}$ obtained by some other method (e.g. Hall effect).

As a conclusion, we would like to emphasize once more that both our calculations and the experiments cited correspond to the case of macroscopic contacts with the dimensions considerably exceeding the characteristic size of inhomogeneities when the measured capacity results from some kind of surface averaging. The real pattern of doping profile could be, in principle, obtained by scanning capacitance microscopy [13]. However, in the geometry of this method the measured local CVC completely differs from $C \sim|V|^{-1 / 2}[14]$ and still has got no adequate theoretical description suitable for extracting quantitative information.

\section{References}

[1] Schroder D K 1998 Semiconductor Material and Device Characterization 2nd edn (New York: Wiley)

[2] Shik A Y 1995 Electronic Properties of Inhomogeneous Semiconductors (London: Gordon and Breach)

[3] Krukovskaya L P, Berman L S, Vul A Y and Shik A Y 1977 Sov. Phys. Semicond $\mathbf{1 1} 1109$

[4] Lebedev A A 1996 Semiconductors 30403

[5] Jones K S and Pearton S J 1984 Phys. Status. Solidi. 82 K101

[6] Patel B K, Nanda K K and Sahu S N 1999 J. Appl. Phys. 85 3666

[7] Konstantinov A O, Harris C I and Janzen E 1994 Appl. Phys. Lett. 652699

[8] Cao F, Oskam G, Searson P C, Stipkala J M, Heimer T A. Farzad F and Meyer G J 1995 J. Phys. Chem. 99 11974

[9] Vanmaekelbergh D, Koster A and Marin F I 1997 Adv. Mater 9575

[10] Jayavel P, Udhayasankar M, Kumar J, Asokan K and Kanjilal D 1999 Nucl. Instrum. Methods B 156110

[11] Abay B, Onganer Y, Saglam M, Efeoglu H, Turut A and Yogurtcu Y K 2000 Microelectr. Eng. 51-52 689

[12] Sullivan J P, Tung R T, Pinto M R and Graham W R 1991 J. Appl. Phys. $\mathbf{7 0} 7403$

[13] Williams C C 1999 Annu. Rev. Mater. Sci. 29471

[14] Shik A and Ruda H E 2002 Surf. Sci. at press 